\title{
A coordinate atlas of the manifold of observable conditioned invariant subspaces
}

\author{
F. Puerta ${ }^{1 *} \quad$ X. Puerta ${ }^{1 \dagger} \quad$ I. Zaballa ${ }^{2 \ddagger}$ \\ ${ }^{1}$ Departament de Matemàtica Aplicada I \\ Universitat Politècnica de Catalunya \\ Diagonal 647, 08028 Barcelona, Spain \\ 2 Departamento de Matemática Aplicada y EIO \\ Universidad del Pais Vasco \\ Apartado 640, 48080 Bilbao, Spain
}

\begin{abstract}
Given an observable system $(C, A) \in \mathbb{K}^{m \times n} \times \mathbb{K}^{n \times n}$ with $\mathbb{K}=\mathbb{R}$ or $\mathbb{C}$, the set of $(C, A)$ invariant subspaces having the restricted system fixed observability indices is a smooth manifold embedded on the corresponding Grassmannian. We obtain an explicit parametrization of it by means of an atlas of coordinate charts. This parametrization can be applied to solve some design problems related to the Disturbance Decoupling Problem.
\end{abstract}

Key words: Conditioned invariant subspace, smooth manifold, coordinate chart, Brunovsky bases, disturbance decoupling problem.

${ }^{*}$ e-mail: puerta@ma1.upc.es. Partially supported by the CAICYT, Proyecto de Investigación PB97-0599CO3-03

†e-mail: coll@ma1.upc.es. Partially supported by the CAICYT, Proyecto de Investigación PB97-0599CO3-03

${ }^{\ddagger}$ e-mail: izaballa@picasso.lc.ehu.es. Partially supported by the CAICYT, Proyecto de Investigación PB97-0599-CO3-01 


\section{Introduction}

Given a finite-dimensional time invariant linear system

$$
\left\{\begin{array}{l}
\dot{x}(t)=A x(t)+B u(t) \\
y(t)=C x(t)
\end{array}\right.
$$

the notions of $(A, B)$-invariant and $(C, A)$-invariant subspaces, or using the terminology of [BM, Ch. 4] $(A, B)$-controlled and $(A, C)$-conditioned subspaces, play a fundamental role in geometric control theory (see for example $[\mathrm{W}])$. The structure of the set of $(C, A)$-invariant subspaces has been studied in the begining 80 's by several authors. The first results on the parametrization of $(C, A)$-invariant subspaces are due to Hinrichsen, Münzer and Prätzel-Wolters ([HMP]) using a module theoretical approach and by Münzer in an unpublished work covering several topological aspects. More recently Fuhrmann and Helmke ([FH] $)$ and Ferrer, F.Puerta and X.Puerta ([FPP1 FPP2]) have made new progress in this area. The last ones study the geometric properties of the set of $(C, A)$-invariant subspaces which are the starting point of this paper. Namely, they prove that this set can be stratified in a finite set of smooth manifolds by fixing the observability indices of the restriction.

Our goal here is to parametrize explicitely these manifolds by means of an atlas of coordinate charts. More precisely, we show that these manifolds can be covered by a finite number of open and dense sets, each one of them being diffeomorphic to $\mathbb{R}^{N}$, where $N$ is the corresponding dimension of the manifold. This kind of parametrization may be useful in several instances of Control Theory. As an example we will show an application closely related to the Disturbane Decoupling Problem (DDP). In fact, it is well-known that this problem has a solution if and only if

$$
\Im(A, B ; \operatorname{Ker} C) \supset \operatorname{Im} Q
$$

where $\Im(A, B$; $\operatorname{Ker} C)$ is the unique maximal $(A, B)$-invariant subspace contained in $\operatorname{Ker} C$ and $Q$ is the disturbing matrix. By duality this is equivalent to

$$
\Im(A, B ; \operatorname{Ker} C)^{\perp} \subset(\operatorname{Im} Q)^{\perp} .
$$

Since $\Im(A, B \text {; Ker } C)^{\perp}$ is a $\left(B^{T}, A^{T}\right)$-invariant subspace, one is interested in the description of the $\left(B^{T}, A^{T}\right)$-invariant subspace contained in a given subspace. We will see in an example that by using the coordinate charts this problem can be reduced to finding the solution of a system of linear equations. We refer also to [WE] where the interest of finding $(A, B)$-invariant subspaces of minimal dimension and containing arbitrary subspaces is pointed out.

The structure of this paper is as follows: In section 2 we introduce the notation and the main basic concepts to be used along the paper. We also present an alternative description to the one given in [FPP1], of the set of $(C, A)$-invariant subspaces as an homogeneous space which is more convenient in order to obtain a collection of coordinate charts. In section 3 we find explicitly an atlas of coordinate charts for the differentiable manifold of $(C, A)$-invariant subspaces. Finally, section 4 contains an example of the application of the coordinate charts to solve a problem of designing an output matrix $C$ for a Decoupling Disturbance Problem to have a solution. 


\section{Notation and Preliminaries}

In this paper we make use of the following notation and background. $\mathbb{K}$ is the field of either the complex or real numbers. $\mathcal{M}_{p, q}$ denotes the set of $p \times q$ matrices with entries in $\mathbb{K}$ and $\mathcal{M}_{p, q}^{*}$ the set of full rank ones. If $p=q$ we write simply $\mathcal{M}_{p}$ and $\mathcal{M}_{p}^{*}$, respectively. We identify $\mathcal{M}_{p}^{*}$ with the general linear group $\mathrm{Gl}\left(\mathbb{K}^{p}\right)$.

$\operatorname{Gr}_{d}(\mathcal{X})$ denotes the Grassman manifold of $d$-dimensional subspaces of a vector space $\mathcal{X}$. We identify a $d$-tuple of vectors of $\mathbb{K}^{n}$ with the $n \times d$-matrix having in the column-entries the coefficients of these vectors. We then identify $\operatorname{Gr}_{d}\left(\mathbb{K}^{n}\right)$ with the orbit space of $\mathcal{M}_{n, d}^{*}$ under the action of $\mathrm{Gl}_{d}(\mathbb{K})$ by right multiplication denoted $\mathcal{M}_{n, d}^{*} / \mathrm{Gl}\left(\mathbb{K}^{d}\right)$. If $X \in \mathcal{M}_{n, d}^{*},[X]$ denote the subspace of $\mathbb{K}^{n}$ spanned by the columns of $X$.

We fix an observable pair $(C, A)$ with $A \in \mathcal{M}_{n}$ and $C \in \mathcal{M}_{r, n}$. There is no lost of generality assuming that $C$ has full rank $r$.

We recall that a subspace $\mathcal{S}$ of $\mathbb{K}^{n}$ is $(C, A)$-invariant if $A(\mathcal{S} \cap \operatorname{Ker} C) \subset \mathcal{S}$, which is equivalent to the existence of an output injection $K \in \mathcal{M}_{n, r}$ such that $(A+K C) \mathcal{S} \subset \mathcal{S}$. We will use this notion in a different but equivalent way more conviniet for our purposes. Let $\mathcal{X}=\mathbb{K}^{n+r}$ and let $\mathcal{Y}$ be the $n$-dimensional subspace spanned by the $n$ first elements of the canonical basis. We identify $\mathbb{K}^{n}$ with $\mathcal{Y}$ via $x \mapsto(x, 0)$. We identify the pair $(C, A)$ with the linear map $f: \mathcal{Y} \longrightarrow \mathcal{X}$ defined by $(x, 0) \mapsto(A x, C x)$. It can be easily seen that a subspace $\mathcal{S}$ of $\mathcal{Y}$ is $(C, A)$-invariant if and only if $f(\mathcal{S}) \cap \mathcal{Y} \subset \mathcal{S}$ (in fact $f^{-1}(\mathcal{Y})$ can be identified with $\operatorname{Ker} C$ ). According to this identification $\mathcal{S}$ is said to be an $f$-invariant subspace.

In general, if $\mathcal{X}$ is any $(n+r)$-dimensional vector space, $\mathcal{Y}$ a $n$-dimensional subspace of $\mathcal{X}$ and $f$ a linear map $f: \mathcal{Y} \longrightarrow \mathcal{X}$, a subspace $\mathcal{S}$ of $\mathcal{Y}$ is called $f$-invariant if the above relation holds; i.e. $f(\mathcal{S}) \cap \mathcal{Y} \subset \mathcal{S}$.

We recall, (see $[\mathrm{FP}]$ for more details) that we can associate to $f$ a Brunovsky canonical form. In fact, if we take a basis of $\mathcal{X}$ obtained by extending a basis of $\mathcal{Y}$, the matrix of $f$ in these bases has the form $\left(\begin{array}{l}A \\ C\end{array}\right)$ with $A \in \mathcal{M}_{n}$ and $C \in \mathcal{M}_{r, n}$. If we change these bases, the new pair is feedback equivalent ([B]) or block-similar ([1]) to $\left(\begin{array}{l}A \\ C\end{array}\right)$, so that it makes sense to define the Brunovsky canonical form of $f$ as the Brunovsky canonical form of $\left(\begin{array}{l}A \\ C\end{array}\right)$, the observability indices of $f$ as those of $\left(\begin{array}{l}A \\ C\end{array}\right)$, etc. With this notation $\mathcal{S}$ is $f$-invariant means that $\left(\begin{array}{l}A \\ C\end{array}\right)$ is feedback equivalent to a system with the form

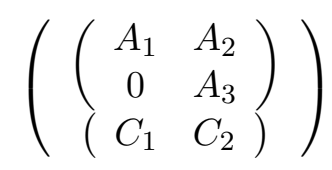

where $\left(\begin{array}{l}A_{1} \\ C_{1}\end{array}\right)$ is a restriction of $\left(\begin{array}{l}A \\ C\end{array}\right)$ to $\mathcal{S}$. Notice that we say "a restriction", no "the restriction", because $\left(A_{1}, C_{1}\right)$ is not uniquely determined. However, all pairs defined in this way are feedback equivalent. 
If $\left(\begin{array}{l}A \\ C\end{array}\right)$ is observable, $f$ is called observable and then for every subspace $\mathcal{S}$ of $\mathcal{Y}$ the restriction $f_{\mid S}: S \longrightarrow \mathcal{X}$ is also observable. As above, we identify the linear map associated to $\left(\begin{array}{l}A \\ C\end{array}\right)$ with the pair $(C, A)$.

Let $\underline{k}=\left(k_{1}, \ldots, k_{r}\right)$ be the observability indices of $(C, A), k_{1} \geq \cdots \geq k_{r}>0$. One can prove the following interpretation of $\underline{k}$ in terms of $f$ (see [FP]). There exists a basis of $\mathcal{Y}$ consisting of vectors

$$
\left.\begin{array}{c}
v_{1}, f\left(v_{1}\right), \ldots, f^{k_{1}-1}\left(v_{1}\right) \\
\ldots \ldots \ldots \ldots \ldots \ldots \ldots \\
v_{r}, f\left(v_{r}\right), \ldots, f^{k_{r}-1}\left(v_{r}\right)
\end{array}\right\}
$$

so that the family $\left(f^{k_{1}}\left(v_{1}\right), \ldots, f^{k_{r}}\left(v_{r}\right)\right)$ completes a basis of $\mathcal{X}$. Notice that the matrix of $f$ with respect to these bases of $\mathcal{Y}$ and $\mathcal{X}$ is the Brunovsky canonical form of $(C, A)$. We then call the basis in (1) a Brunovsky basis of $\mathcal{Y}$ or, shortly, a $B$-basis of $\mathcal{Y}$, and the corresponding extended basis of $\mathcal{X}$, a $B$-basis of $\mathcal{X}$.

Given a partition $\underline{h}=\left(h_{1}, \ldots, h_{s}\right)$ with $h_{1} \geq \ldots \geq h_{s}>0$ compatible with $\underline{k}$ (i. e. $k_{i} \geq h_{i}$ for $1 \leq i \leq s)$ we denote by $\operatorname{Inv}(\underline{k}, \underline{h})$ the set of $f$-invariant subspaces $\mathcal{S}$ such that the restriction $f_{\mid \mathcal{S}}: \mathcal{S} \longrightarrow \mathcal{X}$ has observability indices $h_{1}, \ldots, h_{s}$. Thus a $B$-basis of $\mathcal{S}$ with respect to $f_{\mid \mathcal{S}}$ is of the form:

$$
\left.\begin{array}{c}
u_{1}, f\left(u_{1}\right), \ldots, f^{h_{1}-1}\left(u_{1}\right) \\
\ldots \ldots \ldots \ldots \ldots \ldots \\
u_{s}, f\left(u_{s}\right), \ldots, f^{h_{s}-1}\left(u_{s}\right)
\end{array}\right\}
$$

As proved in [FPP1], the sets Inv $(\underline{k} ; \underline{h})$, are smooth manifolds defining a finite stratification of the set of $f$-invariant subspaces of $\mathcal{Y}$. We now briefly recall the description of $\operatorname{Inv}(\underline{k}, \underline{h})$ as an homogeneous space (see [FPP1]).

We assume that the canonical basis of $\mathcal{X}=\mathbb{K}^{n+r}$ is a $B$-basis (with respect to $f$ ). Then, its first $n$ elements are a $B$-basis of $\mathcal{Y}$.

Let $\mathcal{M}(\underline{k} ; \underline{h})$ be the set of matrices $X \in \mathcal{M}_{n, d}^{*}$ such that $[X] \in \operatorname{Inv}(\underline{k} ; \underline{h})$ and their columns are a $B$-bases of $[X]$ (whith respect to $f_{\mid[X]}$ ). This set of matrices is characterized as the solutions of certain matrix equations and they have a description as block upper Toeplitz matrices ([FPP1, Prop.3.4]). We denote $\mathcal{G}(\underline{h}):=\mathcal{M}(\underline{h} ; \underline{h})$.

It was proved in [FPP1, Th. 4.5] that $\mathcal{G}(\underline{h})$ is a subgroup of $\mathrm{Gl}\left(\mathbb{K}^{d}\right)$ acting freely on $\mathcal{M}(\underline{k} ; \underline{h})$ from the right by matrix multiplication and that the orbit space $\mathcal{M}(\underline{k} ; \underline{h}) / \mathcal{G}(\underline{h})$ is a smooth manifold. From this, a differentiable structure in Inv $(\underline{k} ; \underline{h})$ is defined through the bijection $\mathcal{M}(\underline{k} ; \underline{h}) / \mathcal{G}(\underline{h}) \longrightarrow \operatorname{Inv}(\underline{k} ; \underline{h})$.

Our goal is to obtain an atlas of coordinate charts for Inv $(\underline{k} ; \underline{h})$; and for this it is more convenient to provide Inv $(\underline{k} ; \underline{h})$ with another representation as a quotient manifold.

Put $k=k_{1}, h=h_{1}$ and let $\underline{r}=\left(r_{1}, \ldots, r_{k}\right)$ with $r=r_{1} \geq r_{2} \geq \ldots \geq r_{k}>0$ and $\underline{s}=\left(s_{1}, \ldots, s_{h}\right)$ with $s=s_{1} \geq \ldots \geq s_{h}>0$ be the conjugate partitions of $\underline{k}$ and $\underline{h}$ respectively. For notational 
convenience we will agree that $r_{i}:=0$ for $i>k$ and $s_{i}:=0$ for $i>h$. The positive integers $r_{1} \geq \ldots \geq r_{k}$ are called the Brunovsky indices of $\left(\begin{array}{l}A \\ C\end{array}\right)$ or Brunovsky indices of $f$ (see [B]).

We reorder the fixed $B$-basis of $\mathcal{Y}$ as follows:

$$
\left.\begin{array}{l}
f^{k_{1}-1}\left(v_{1}\right), \ldots, f^{k_{r}-1}\left(v_{r}\right) \\
\ldots \ldots \ldots \ldots \ldots \ldots \ldots \ldots \\
f^{k_{1}-i}\left(v_{1}\right), \ldots, f^{k_{r_{i}}-i}\left(v_{r_{i}}\right) \\
v_{1}, \ldots, v_{r_{k}}
\end{array}\right\}
$$

We reorder also the $B$-basis of the elements of $\operatorname{Inv}(\underline{k}, \underline{h})$ in the same way (replace in (3) $k_{i}$ and $r_{i}$ by $h_{i}$ and $s_{i}$, respectively). Notice that, by definition of $r_{i}$ and $s_{i}, k_{r_{i}} \geq i$ and $h_{s_{i}} \geq i$. We call $B^{*}$-bases these reordered $B$-bases.

Once a $B^{*}$-basis of $\mathcal{Y}$ has been fixed we can identify the vectors of $\mathcal{Y}$ with their components with respect to the fixed basis. Bearing in mind this observation we introduce the sets $\mathcal{M}(\underline{r}, \underline{s})$ and $\mathcal{G}(\underline{s})$ :

Definition 2.1 $\mathcal{M}(\underline{r}, \underline{s})$ is the set of matrices $X \in \mathcal{M}_{n, d}^{*}$ such that

1. $[X] \in \operatorname{Inv}(\underline{k} ; \underline{h})$.

2. The columns of $X$ are the components of a $B^{*}$-basis of $[X]$ with respect to (3).

We denote $\mathcal{G}(\underline{s}):=\mathcal{M}(\underline{s}, \underline{s})$.

We will need a precise description of the elements of $\mathcal{M}(\underline{r} ; \underline{s})$. We next illustrate them with an example.

Example 2.2 Suppose $\underline{k}=(4,3,2,2,1)$ and $\underline{h}=(3,2,2)$. Then $\underline{r}=(5,4,2,1)$ and $\underline{s}=$ $(3,3,1)$. It was shown in [FPP1] that a matrix $X \in \mathcal{M}(\underline{k} ; \underline{h})$ has the form

$$
X=\left(\begin{array}{ccc|cc|cc}
x_{1} & 0 & 0 & y_{1} & 0 & z_{1} & 0 \\
x_{2} & x_{1} & 0 & y_{2} & y_{1} & z_{2} & z_{1} \\
0 & x_{2} & x_{1} & y_{3} & y_{2} & z_{3} & z_{2} \\
0 & 0 & x_{2} & 0 & y_{3} & 0 & z_{3} \\
\hline t_{1} & 0 & 0 & u_{1} & 0 & v_{1} & 0 \\
0 & t_{1} & 0 & u_{2} & u_{1} & v_{2} & v_{1} \\
0 & 0 & t_{1} & 0 & u_{2} & 0 & v_{2} \\
\hline 0 & 0 & 0 & w_{1} & 0 & m_{1} & 0 \\
0 & 0 & 0 & 0 & w_{1} & 0 & m_{1} \\
\hline 0 & 0 & 0 & p_{1} & 0 & q_{1} & 0 \\
0 & 0 & 0 & 0 & p_{1} & 0 & q_{1} \\
\hline 0 & 0 & 0 & 0 & 0 & 0 & 0
\end{array}\right)
$$


and it corresponds to the components of the $B$-basis

$$
\left\{u_{1}, f\left(u_{1}\right), f^{2}\left(u_{1}\right), u_{2}, f\left(u_{2}\right), u_{3}, f\left(u_{3}\right)\right\}
$$

of the $f$-invariant subspace $\mathcal{S}$ with respect to the $B$-basis

$$
\left\{v_{1}, f\left(v_{1}\right), f^{2}\left(v_{1}\right), f^{3}\left(v_{1}\right), v_{2}, f\left(v_{2}\right), f^{2}\left(v_{2}\right), v_{3}, f\left(v_{3}\right), v_{4}, f\left(v_{4}\right), v_{5}\right\}
$$

of $\mathcal{Y}$. Then, we reorder these bases as indicated above:

$$
\left\{f^{2}\left(u_{1}\right), f\left(u_{2}\right), f\left(u_{3}\right), f\left(u_{1}\right), u_{2}, u_{3}, u_{1}\right\}
$$

for $\mathcal{S}$ and

$$
\left\{f^{3}\left(v_{1}\right), f^{2}\left(v_{2}\right), f\left(v_{3}\right), f\left(v_{4}\right), v_{5}, f^{2}\left(v_{1}\right), f\left(v_{2}\right), v_{3}, v_{4}, f\left(v_{1}\right), v_{2}, v_{1}\right\}
$$

for $\mathcal{Y}$ and we get

$$
\begin{aligned}
& f^{2}\left(u_{1}\right)=x_{1} f^{2}\left(v_{1}\right)+x_{2} f^{3}\left(v_{1}\right)+t_{1} f^{2}\left(v_{2}\right) \\
& f\left(u_{2}\right)=y_{1} f\left(v_{1}\right)+y_{2} f^{2}\left(v_{1}\right)+y_{3} f^{3}\left(v_{1}\right)+u_{1} f\left(v_{2}\right)+u_{2} f^{2}\left(v_{2}\right)+w_{1} f\left(v_{3}\right)+p_{1} f\left(v_{4}\right) \\
& \text { etc. }
\end{aligned}
$$

The matrix representation of $\mathcal{S}$ with respect to these new bases is

$$
\left(\begin{array}{ccc|ccc|c}
x_{2} & y_{3} & z_{3} & & & \\
t_{1} & u_{2} & v_{2} & & & \\
0 & w_{1} & m_{1} & & \mathbf{0} & \mathbf{0} \\
0 & p_{1} & q_{1} & & & & \\
0 & 0 & 0 & & & & \\
\hline x_{1} & y_{2} & z_{2} & x_{2} & y_{3} & z_{3} & \\
0 & u_{1} & v_{1} & t_{1} & u_{2} & v_{2} & \mathbf{0} \\
0 & 0 & 0 & 0 & w_{1} & m_{1} & \\
0 & 0 & 0 & 0 & p_{1} & q_{1} & \\
\hline 0 & y_{1} & z_{1} & x_{1} & y_{2} & z_{2} & x_{2} \\
0 & 0 & 0 & 0 & u_{1} & v_{1} & t_{1} \\
\hline 0 & 0 & 0 & 0 & y_{1} & z_{1} & x_{1}
\end{array}\right)
$$

This is the form of the matrices in $\mathcal{M}(\underline{r}, \underline{s})$. Notice that they are lower block triangular matrices and that the first block column determines the rest of the matrix.

Remark 2.3 It is clear that $X \in \mathcal{M}(\underline{r}, \underline{s})$ if and only if $V X U^{-1} \in \mathcal{M}(\underline{k}, \underline{h})$ for certain permutation matrices $U$ and $V$.

The following lemma gives explicitly the form of the matrices $U$ and $V$. 
Lemma 2.4 If we partition $U$ into blocks, according to the partitions $s_{1} \geq \ldots \geq s_{h}$ and $h_{1} \geq \ldots \geq h_{s}$

$$
U=\left(\begin{array}{cccc}
U_{11} & U_{12} & \ldots & U_{1 h} \\
\vdots & \vdots & & \vdots \\
U_{s 1} & U_{s 2} & \ldots & U_{s h}
\end{array}\right)
$$

then, $U_{i j}$ is a $h_{i} \times s_{j}$ matrix such that if $h_{i} \geq j$ then its entries are all zero but the element in the $\left(h_{i}-j+1\right)$ st row and ith column, which is 1 , and $U_{i j}$ is the zero matrix if $h_{i}<j$. An analogous description for $V$ is obtained replacing $s$ and $h$ by $r$ and $k$.

We now give without proof a general description of $\mathcal{M}(\underline{r}, \underline{s})$ and $\mathcal{G}(\underline{s})$ :

Proposition 2.5 A matrix $X \in \mathcal{M}_{n, d}$ belongs to $\mathcal{M}(\underline{r}, \underline{s})$ if and only if $X$ can be partitioned into blocks $X=\left(X_{i j}\right), 1 \leq i \leq k, 1 \leq j \leq h$ in such a way that

(i) $X_{i j} \in \mathcal{M}_{r_{i}, s_{j}}$.

(ii) $X_{i j}=0$ if $i<j$.

(iii) If $i \geq j, X_{i j}$ can be partitioned into blocks $X_{i j}=\left(Z_{i \alpha}^{i-j+1}\right), 1 \leq \alpha \leq h-j+1$, where

$$
Z_{i \alpha}^{i-j+1}=\left(\begin{array}{c}
Y_{i \alpha}^{i-j+1} \\
\mathbf{0}
\end{array}\right)
$$

with $Y_{i \alpha}^{i-j+1}$ having size $r_{h-\alpha+i-j+1} \times\left(s_{h-\alpha+1}-s_{h-\alpha+2}\right)$.

(iv) $X_{i+1, j+1}$ is obtained from $X_{i j}$ by removing the last $s_{j}-s_{j+1}$ columns and the last $r_{i}-r_{i+1}$ rows for $1 \leq i \leq k, 1 \leq j \leq h$.

(v) $\operatorname{rank} X_{i i}=s_{i}, 1 \leq i \leq k$.

We call the block decomposition of $X_{i j}$ in (iii), its standard block decomposition.

Remark 2.6 From (iv) it can be easily seen that for $i=1, \ldots, h X_{i i}$, and then $X$, have maximal rank if and only if $X_{11}$ has maximal rank. (See also [FPP1, Prop. 3.7]).

In order to describe the matrices of $\mathcal{G}(\underline{s})$ we only have to replace $\underline{k}$ and $\underline{r}$ by $\underline{h}$ and $\underline{s}$ in the above proposition. Nevertheless a better description is provided by the following proposition whose proof is a straightforward conclusion of the previous one.

Proposition 2.7 .- A matrix $T \in \mathcal{M}_{d, d}^{*}$ belongs to $\mathcal{G}(\underline{s})$ if and only if $T$ can be partitioned into blocks $T=\left(T_{i j}\right)_{1 \leq i, j \leq h}$ such that 
(i) $T_{i j} \in \mathcal{M}_{s_{i}, s_{j}}$.

(ii) For $i<j, T_{i j}=0$.

(iii) For $1 \leq i \leq h, T_{i i}=\left(\begin{array}{cccc}T_{11}^{1} & T_{12}^{1} & \ldots & T_{1, h-i+1}^{1} \\ 0 & T_{22}^{1} & \cdots & T_{2, h-i+1}^{1} \\ \vdots & \vdots & \ddots & \vdots \\ 0 & 0 & \ldots & T_{h-i+1, h-i+1}^{1}\end{array}\right)$, with $T_{j j}^{1}$ invertible of size $\left(s_{h-j+1}-s_{h-j+2}\right), 1 \leq j \leq h-i+1$.

(iv) For $i>j, T_{i j}=\left(\begin{array}{ccccccc}0 & \ldots & 0 & T_{1 i-j+1}^{i-j+1} & T_{1, i-j+2}^{i-j+1} & \ldots & T_{1, h-j+1}^{i-j+1} \\ 0 & \ldots & 0 & 0 & T_{2, i-j+2}^{i-j+1} & \ldots & T_{2, h-j+1}^{i-j+1} \\ \vdots & \ldots & \vdots & \vdots & \vdots & \ddots & \vdots \\ 0 & \ldots & 0 & 0 & 0 & \ldots & T_{h-i+1, h-j+1}^{i-j+1}\end{array}\right)$, with $T_{\alpha \beta}^{i-j+1}$ is of size $\left(s_{h-\alpha+1}-s_{h-\alpha+2}\right) \times\left(s_{h-\beta+1}-s_{h-\beta+2}\right)$.

As we said above, a differentiable structure in Inv $(\underline{k} ; \underline{h})$ was introduced in [FPP1] through the natural bijection between this set and the orbit space $\mathcal{M}(\underline{k} ; \underline{h}) / \mathcal{G}(\underline{h})$. The following theorem gives an alternative description of $\operatorname{Inv}(\underline{k} ; \underline{h})$ as a homogeneous spaces.

Theorem 2.8 With the above notation

(i) $\mathcal{G}(\underline{s})$ is a subgroup of $\mathrm{Gl}\left(\mathbb{K}^{d}\right)$.

(ii) $\mathcal{G}(\underline{s})$ acts freely on $\mathcal{M}(\underline{r}, \underline{s})$ on the right by matrix multiplication.

(iii) If $X \in \mathcal{M}(\underline{r}, \underline{s}), T \in \mathrm{Gl}\left(\mathbb{K}^{d}\right)$ and $X T \in \mathcal{M}(\underline{r}, \underline{s})$, then $T \in \mathcal{G}(\underline{s})$.

(iv) The orbit space $\mathcal{M}(\underline{r}, \underline{s}) / \mathcal{G}(\underline{s})$ has a differentiable structure such that the natural projection $\mathcal{M}(\underline{r}, \underline{s}) \longrightarrow \mathcal{M}(\underline{r} ; \underline{s}) / \mathcal{G}(\underline{s})$ is a submersion.

(v) The map $X \longmapsto V X U^{-1}$ defined in (2.3) induces a diffeormorphism from $\mathcal{M}(\underline{r}, \underline{s}) / \mathcal{G}(\underline{s})$ into $\mathcal{M}(\underline{k} ; \underline{h}) / \mathcal{G}(\underline{h})$.

Proof . (i), (ii) and (iii) follows from Remark 2.3 and [FPP1, Prop. 3.9 and Lemma 4.4]. Then in an analogous way to [FPP1, Th. 4.5] we conclude that (iv) is true. Finally, since the map $X \longmapsto V X U^{-1}$ is a diffeormorphism and the corresponding natural projections have local sections, then (v) follows.

Since there is a natural bijection between $\operatorname{Inv}(\underline{k} ; \underline{h})$ and $\mathcal{M}(\underline{k}, \underline{h}) / \mathcal{G}(\underline{h})$ (see [FPP1, Cor. 3.3]) we also have a natural bijection between $\operatorname{Inv}(\underline{k} ; \underline{h})$ and $\mathcal{M}(\underline{r}, \underline{s}) / \mathcal{G}(\underline{s})$. From now on, we consider in Inv $(\underline{k} ; \underline{h})$ the differentiable structure defined through this bijection. 
Corollary 2.9 $\operatorname{dim} \operatorname{Inv}(\underline{k} ; \underline{h})=\sum_{i=1}^{h} \sum_{j=1}^{k} m_{i}\left(r_{i+j-1}-s_{i+j-1}\right)$, where $m_{j}=s_{j}-s_{j+1}$.

Proof . According to Proposition 2.5 the only independent parameters of $X \in \mathcal{M}(\underline{r}, \underline{s})$ are those of $X_{i 1}, 1 \leq i \leq k$, and by the same proposition this number is equal to

$$
m_{1} r_{i}+\ldots+m_{h} r_{h+i-1}=\sum_{j=1}^{h} m_{j} r_{i+j-1}
$$

Then, the corollary follows immediately.

In the next section we will parametrize $\mathcal{M}(\underline{r} ; \underline{s}) / \mathcal{G}(\underline{s})$ by a system of coordinate charts. Those charts are obtained as local sections of the projection

$$
\mathcal{M}(\underline{r} ; \underline{s}) \longrightarrow \mathcal{M}(\underline{r} ; \underline{s}) / \mathcal{G}(\underline{s})
$$

of the principal bundle defined by the corresponding group action. Roughly speaking we obtain a canonical representative for each orbit in $\mathcal{M}(\underline{r} ; \underline{s})$.

Remark 2.10 Going back to Propositions 2.5 and 2.7, we notice that matrix $T_{11}$ is nonsingular and acts on $X_{11}$. Let $\mathcal{B}(\underline{r}, \underline{s})$ and $\mathcal{G B}(\underline{s})$ denote the sets of full rank matrices of the types $X_{11}$ and $T_{11}$, respectively. It is not difficult to see that there is a bijective map between the orbit space $\mathcal{B}(\underline{r}, \underline{s}) / \mathcal{G B}(\underline{s})$ and the generalized flag manifold studied in [HS] (see also [PH] for some additional properties of this manifold). Thus, as a by-product of our main results we will obtain a coordinate atlas for the generalized flag manifold studied in [HS].

\section{An atlas of coordinate charts of $\operatorname{Inv}(\underline{k} ; \underline{h})$}

The coordinate charts of Inv $(\underline{k} ; \underline{h})$ are obtained as a system of canonical representatives of the orbits of its homogeneous space description $\mathcal{M}(\underline{r}, \underline{s}) / \mathcal{G}(\underline{s})$. The algorithm for reducing an element of $\mathcal{M}(\underline{r}, \underline{s})$ to a canonical form is based on a sequence of elementary transformations defined by some subsets of $\mathcal{G}(\underline{s})$. We first describe these subsets for the matrices of $\mathcal{G}(\underline{s})$ correspondig to Example 2.2 and illustrate how the action of these elementary matrices can be used to reduce matrix $X$ in this example to a reduced form.

Let then $X$ be the matrix in (4). A general matriz $T \in \mathcal{G}(\underline{s})$ acting on this matrix has the following form:

$$
T=\left(\begin{array}{cc|cc|c}
T_{11}^{1} & T_{12}^{1} & & & \\
0 & T_{22}^{1} & & & \\
\hline 0 & T_{12}^{2} & T_{11}^{1} & T_{12}^{1} & \\
0 & 0 & 0 & T_{22}^{1} & \\
\hline 0 & 0 & 0 & T_{12}^{2} & T_{11}^{1}
\end{array}\right)
$$


where $T_{11}^{1}$ is $1 \times 1$ and $T_{22}^{1}$ is $2 \times 2$. The following type of matrices related to $T$ will be called elementary in the next definition:

1. $P=\operatorname{diag}\left(P_{11}, P_{22}, P_{33}\right)$ where $P_{11}=\operatorname{diag}\left(Q_{1}, Q_{2}\right), P_{22}=\operatorname{diag}\left(Q_{1}, Q_{2}\right)$ and $P_{33}=$ $\operatorname{diag}\left(Q_{1}\right), Q_{1}, Q_{2}, Q_{3}$ being invertible matrices. (In this case $P_{11}=P_{22}$ because $m_{3}=0$, but in general $P_{i i}$ would have more diagonal blocks than $\left.P_{i+1 i+1}\right)$. The subgroup of $\mathcal{G}(\underline{s})$ of this kind of block diagonal matrices wil be denoted by $\mathcal{G}_{I}(\underline{s})$

2. $E=\operatorname{diag}\left(E_{12}^{1}, E_{12}^{2}, I_{1}\right)$ where

$$
E_{12}^{1}=\left(\begin{array}{cc}
I_{1} & Q \\
0 & I_{2}
\end{array}\right) \text { and } E_{12}^{2}=\left(\begin{array}{cc}
I_{1} & Q \\
0 & I_{2}
\end{array}\right)
$$

(Again $E_{12}^{1}=E_{12}^{2}$ because $m_{3}=0$ but in general $E_{12}^{1}$ and $E_{12}^{2}$ would have different sizes). $\mathcal{G}_{I I}(\underline{s})$ will denote the subgroup of $\mathcal{G}(\underline{s})$ of these block diagonal matrices.

3. $F_{2}=\left(\begin{array}{ccc}I_{3} & 0 & 0 \\ Q_{21} & I_{3} & 0 \\ 0 & Q_{32} & I_{1}\end{array}\right)$ where $Q_{21}=\left(\begin{array}{cc}0 & R_{12}^{1} \\ 0 & 0\end{array}\right)$ and $Q_{32}=\left(\begin{array}{ll}0 & R_{12}^{1}\end{array}\right)$. We will denote by $\mathcal{G}_{I I I}(\underline{s})$ the subgroup of $\mathcal{G}(\underline{s})$ formed by these lower block triangular matrices.

It is clear that any product of matrices of these three types is in $\mathcal{G}(\underline{s})$ and conversely, any matrix in $\mathcal{G}(\underline{s})$ may written as a product of matrices of these three types.

Before going into formal definitions and proofs let us bring matrix $X$ in (4) to a reduced from by using the above elementary matrices. Since $\operatorname{rank} X_{11}=3$ we must have that $x_{2} \neq 0$ or $t_{1} \neq 0$. Assume that $x_{2} \neq 0$ and put $T_{11}^{1}=\left[1 / x_{2}\right], P_{11}=\operatorname{diag}\left(T_{11}^{1}, I_{2}\right), P_{22}=\operatorname{diag}\left(T_{11}^{1}, I_{2}\right), P_{33}=T_{11}^{1}$ and $P_{1}=\operatorname{diag}\left(P_{11}, P_{22}, P_{33}\right)$. Then $P_{1} \in \mathcal{G}_{I}(\underline{s})$ and

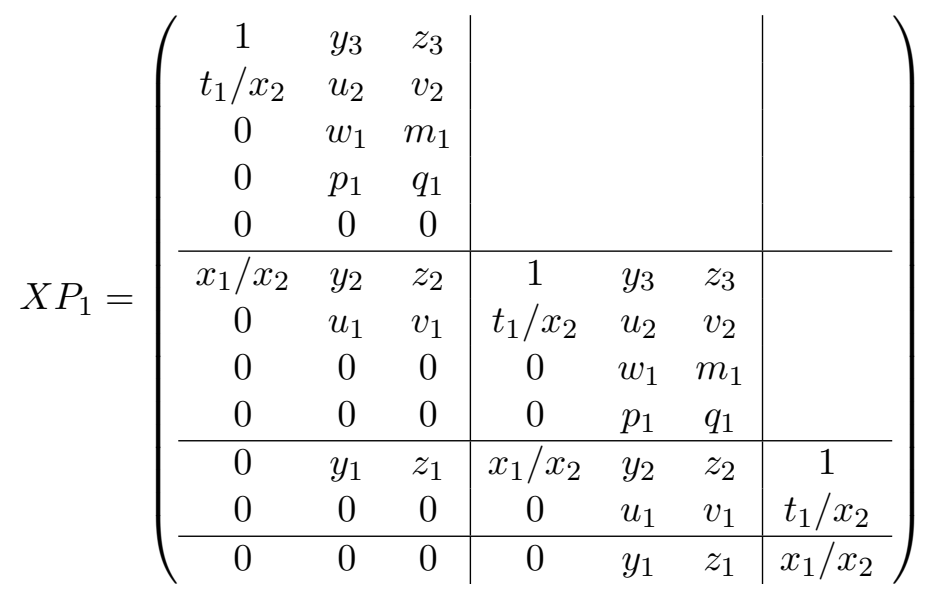

Now, with the 1 in position $(1,1)$ of each diagonal block we can make zero the entries $y_{3}$ and $z_{3}$. This is equivalent to multiplying $X P_{1}$ by a matrix of the form $P_{2}=\operatorname{diag}\left(E_{12}^{1}, E_{12}^{2}, I_{1}\right) \in \mathcal{G}_{I I}(\underline{s})$ 
with $Q=\left[\begin{array}{ll}-y_{3} & -z_{3}\end{array}\right]$. The new block $(1,1)$ is

$$
\left(\begin{array}{ccc}
1 & 0 & 0 \\
t_{1}^{\prime} & u_{2}^{\prime} & v_{2}^{\prime} \\
0 & w_{1} & m_{1} \\
0 & p_{1} & q_{1} \\
0 & 0 & 0
\end{array}\right)
$$

that must have rank 3. So, two rows of $\left(\begin{array}{cc}u_{2}^{\prime} & v_{2}^{\prime} \\ w_{1} & m_{1} \\ p_{1} & q_{1} \\ 0 & 0\end{array}\right)$ must be linearly independent. Assume that, for example, the first and the third ones are linearly independent. Then there is a matrix $P_{3} \in \mathcal{G}_{I}(\underline{s})$ such that

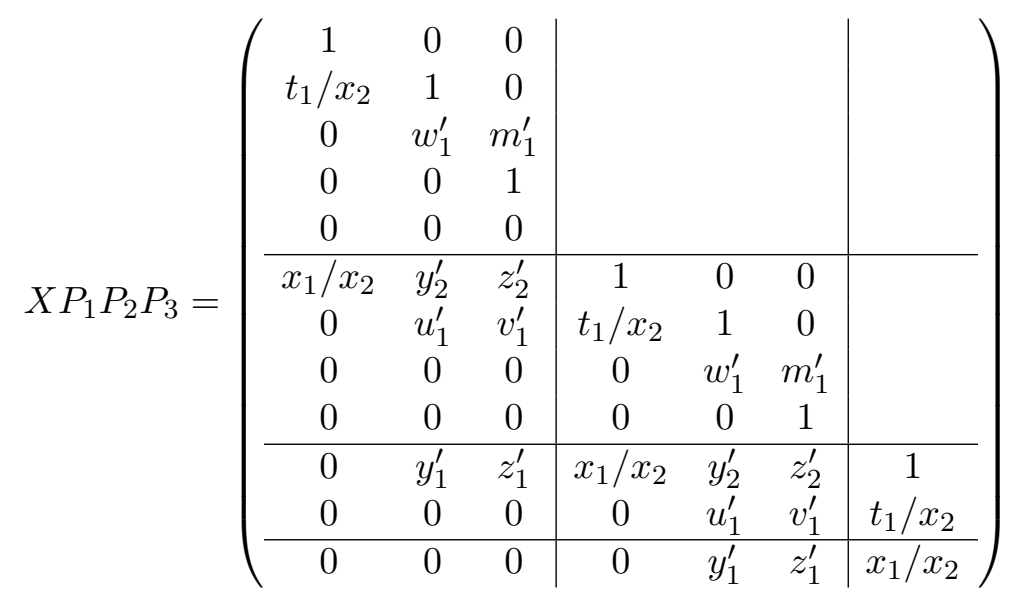

Finally we proceed to make zero as many entries of the nondiagonal blocks as possible. This can be only made with the third type of elementary matrices. In this case

$$
F_{2}=\left(\begin{array}{ccc}
I_{3} & 0 & 0 \\
Q_{21} & I_{3} & 0 \\
0 & Q_{32} & I_{1}
\end{array}\right)
$$

with $Q_{21}=\left(\begin{array}{cc}0 & R_{12}^{1} \\ 0 & 0\end{array}\right)$ and $Q_{32}=\left(\begin{array}{ll}0 & R_{12}^{1}\end{array}\right)$. So we can only make zero the elements $y_{2}^{\prime}$ 
and $z_{2}^{\prime}$ and obtain the following matrix

$$
\left(\begin{array}{lll|lll|l}
1 & 0 & 0 & & & & \\
a & 1 & 0 & & & & \\
0 & b & c & & & & \\
0 & 0 & 1 & & & & \\
0 & 0 & 0 & & & & \\
\hline d & 0 & 0 & 1 & 0 & 0 & \\
0 & e & f & a & 1 & 0 & \\
0 & 0 & 0 & 0 & b & c & \\
0 & 0 & 0 & 0 & 0 & 1 & \\
\hline 0 & g & h & d & 0 & 0 & 1 \\
0 & 0 & 0 & 0 & e & f & a \\
\hline 0 & 0 & 0 & 0 & g & h & d
\end{array}\right)
$$

No further entries can be made zero by the action of a matrix of $\mathcal{G}(\underline{s})$. A matrix with this form will be called a matrix in reduced form. We will follow the same pattern to deal with the general case.

\section{Definition 3.1 .-}

1. For $\alpha=1,2, \ldots, h$, let $Q_{\alpha}$ be an $m_{h-\alpha+1} \times m_{h-\alpha+1}$ non singular matrix and

$$
P_{i i}=\operatorname{diag}\left(Q_{1}, Q_{2}, \ldots, Q_{h-i+1}\right), \quad 1 \leq i \leq h
$$

We will write $\mathcal{G}_{I}(s)$ to denote the following set of matrices:

$$
\mathcal{G}_{I}(s)=\left\{P ; P=\operatorname{diag}\left(P_{11}, P_{22}, \ldots, P_{h h}\right)\right\} .
$$

2. Let $i \in\{1,2, \ldots, h\}$, and let $E_{\alpha \beta}^{i}(Q)$ be an $s_{i} \times s_{i}$ matrix with the following form:

$$
E_{\alpha \beta}^{i}(Q)=\left(\begin{array}{ccccccc}
I_{m_{h}} & \ldots & 0 & \ldots & 0 & \ldots & 0 \\
\vdots & \ddots & \vdots & & \vdots & & \vdots \\
0 & \ldots & I_{m_{h-\alpha+1}} & \ldots & Q & \ldots & 0 \\
\vdots & & \vdots & \ddots & \vdots & & \vdots \\
0 & \ldots & 0 & \ldots & I_{m_{h-\beta+1}} & \ldots & 0 \\
\vdots & & \vdots & & \vdots & \ddots & \vdots \\
0 & \ldots & 0 & \ldots & 0 & \ldots & I_{m_{i}}
\end{array}\right)
$$

where $Q \in \mathcal{M}_{m_{h-\alpha+1}, m_{h-\beta+1}}$ and $1 \leq \alpha<\beta \leq h-i+1$.

Then $\mathcal{G}_{I I}(\underline{s})$ will denote the following set of matrices

$$
\mathcal{G}_{I I}(\underline{s})=\left\{P ; P=\operatorname{diag}\left(E_{\alpha \beta}^{1}(Q), \ldots, E_{\alpha \beta}^{i}(Q), I_{s_{i+1}}, \ldots, I_{s_{h}}\right)\right\}
$$


3. For $i=2,3, \ldots, h$ and $1 \leq j<i \leq h$ let $Q_{i j}$ be an $s_{i} \times s_{j}$ matrix partitioned into blocks $Q_{i j}=\left(R_{\alpha \beta}^{i-j+1}\right), 1 \leq \alpha \leq h-i+1,1 \leq \beta \leq h-j+1$ such that $R_{\alpha \beta}^{i-j+1}=0$ if $\alpha+i-j>\beta$ and $R_{\alpha \beta}^{i-j+1}$ has size $m_{h-\alpha+1} \times m_{h-\beta+1}$. That is to say

$$
Q_{i j}=\left(\begin{array}{ccccccc}
0 & \ldots & 0 & R_{1, i-j+1}^{i-j+1} & \ldots & \ldots & R_{1, h-j+1}^{i-j+1} \\
0 & \ldots & 0 & 0 & R_{2, i-j+2}^{i-j+1} & \ldots & R_{2, h-j+1}^{i-j+1} \\
\vdots & & \vdots & \vdots & \vdots & \ddots & \vdots \\
0 & \ldots & 0 & 0 & 0 & \ldots & R_{h-i+1, h-j+1}^{i-j+1}
\end{array}\right)
$$

Now, put

$$
F_{i}=\left(\begin{array}{ccccccccc}
I_{s_{1}} & 0 & \ldots & 0 & 0 & \ldots & 0 & \ldots & 0 \\
0 & I_{s_{2}} & \ldots & 0 & 0 & \ldots & 0 & \ldots & 0 \\
\vdots & \vdots & \ddots & \vdots & \vdots & & \vdots & & \vdots \\
Q_{i 1} & 0 & \ldots & I_{s_{i}} & 0 & \ldots & 0 & \ldots & 0 \\
0 & Q_{i+1,2} & \ldots & 0 & I_{s_{i+1}} & \ldots & 0 & \ldots & 0 \\
\vdots & \vdots & \ddots & \vdots & \vdots & \ddots & \vdots & & \vdots \\
0 & 0 & \ldots & Q_{2 i-1, i} & 0 & \ldots & I_{s_{h-i+1}} & \ldots & 0 \\
0 & 0 & \ldots & 0 & Q_{2 i, i+1} & \ldots & 0 & \ldots & 0 \\
\vdots & \vdots & & \vdots & \vdots & \ddots & \vdots & \ddots & \vdots \\
0 & 0 & \ldots & 0 & 0 & \ldots & Q_{h, h-i+1} & \ldots & I_{s_{h}}
\end{array}\right)
$$

and let $\mathcal{G}_{I I I}(\underline{s})$ denote the set of matrices

$$
\mathcal{G}_{I I I}(\underline{s})=\left\{P ; P=F_{i} \text { for some } i, 2 \leq i \leq h\right\}
$$

Matrices belonging to $\mathcal{G}_{I}(\underline{s}), \mathcal{G}_{I I}(\underline{s}), \mathcal{G}_{I I I}(\underline{s})$ will be called elementary matrices of type $I, I I$ and $I I I$, respectively. It is clear that all of them belong to $\mathcal{G}(\underline{s})$.

The following lemma describes how a matrix is transformed under right multiplication by an elementary matrix. We omit the easy proof.

Lemma 3.2 The action of the elements of $\mathcal{G}_{I}(\underline{s}), \mathcal{G}_{I I}(\underline{s})$ and $\mathcal{G}_{I I I}(\underline{s})$ on $\mathcal{M}(\underline{r}, \underline{s})$ on the right can be described as follows: Let $X \in \mathcal{M}(\underline{r}, \underline{s})$ and let $X_{i j}$ denote the $(i, j)$ block of $X$ as in Proposition 2.5. Then

1. If $P \in \mathcal{G}_{I}(\underline{s})$,

$$
(X P)_{i j}=\left(Z_{i 1}^{i-j+1} Q_{1} \cdots Z_{i, h-j+1}^{1-j+1} Q_{h-j+1}\right), \quad 1 \leq i \leq h, \quad 1 \leq j \leq k .
$$


2. If $P=E(\alpha, \beta ; i) \in \mathcal{G}_{I I}(\underline{s})$,

$$
\begin{aligned}
(X P)_{j j}= & \left(Z_{j 1}^{1} \cdots Z_{j \alpha}^{1} \cdots Z_{j \beta}^{1}+Z_{j \alpha}^{1} Q \cdots Z_{j h-j+1}^{1}\right) \\
& 1 \leq j \leq i, 1 \leq \alpha<\beta \leq h-j+1 \\
(X P)_{j j}= & X_{j j}, \quad i+1 \leq j \leq h \\
(X P)_{j t}= & X_{j t}, \quad \text { if } j \neq t
\end{aligned}
$$

3. If $P=F_{i} \in \mathcal{G}_{I I I}(\underline{s})$ and we write $X_{j}=\left(\begin{array}{c}X_{1 j} \\ \vdots \\ X_{k j}\end{array}\right), 1 \leq j \leq h$ and $X=\left(X_{1} \cdots X_{h}\right)$, then

$$
X P=\left(X_{1}+X_{i} Q_{i 1} \cdots X_{h-i+1}+X_{h} Q_{h h-i+1}, X_{h-i+2} \cdots X_{h}\right)
$$

The following theorem shows how to obtain special representatives of each one of the orbits of $\mathcal{M}(\underline{r}, \underline{s}) / \mathcal{G}(\underline{s})$ by using the elementary transformations introduced in Definition 3.1. We will show later that these representatives define, actually, a system of coordinate charts.

Theorem 3.3 For every $X \in \mathcal{M}(\underline{r}, \underline{s})$ there exists a set of positive integers pairwise different $\left\{n_{i j}, 1 \leq i \leq h, 1 \leq j \leq m_{h-i+1}\right\}$ such that for $i=1,2, \ldots, h$

$$
1 \leq n_{i 1}<n_{i 2}<\ldots<n_{i m_{h-i+1}} \leq r_{h-i+1}
$$

and a matrix $P \in \mathcal{G}(\underline{s})$ such that the matrix $Y=X P$ can be partitioned into blocks $Y=\left(Y_{i j}\right)$, $1 \leq i \leq k, 1 \leq j \leq h$, satisfying the following conditions:

(i) $Y_{i j} \in \mathcal{M}_{r_{i}, s_{j}}$.

(ii) $Y_{i j}=0$ if $i<j$.

(iii) For $i=1,2, \ldots, h, Y_{i i}$ can be partitioned into blocks $Y_{i i}=\left(L_{i \beta}^{1}\right), 1 \leq \beta \leq h-i+1$ in such a way that for $\beta=1,2, \ldots, h-i+1, L_{i \beta}^{1} \in \mathcal{M}_{r_{i}, m_{h-\beta+1}}$ is a matrix whose last $r_{i}-r_{h-\beta+1}$ rows are zero, the rows $n_{i j}, 1 \leq i \leq \beta-1,1 \leq j \leq m_{h-i+1}$ are also zero, and the rows $n_{\beta 1}, n_{\beta 2}, \ldots, n_{\beta m_{h-\beta+1}}$ are unit vectors $e_{1}^{\beta}, e_{2}^{\beta}, \ldots, e_{m_{h-\beta+1}}^{\beta}$ :

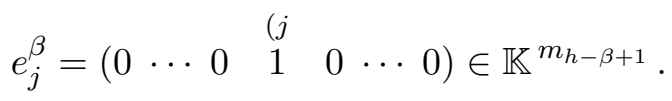

(iv) For $i>j, Y_{i j}$ can be partitioned into blocks $Y_{i j}=\left(L_{i \beta}^{i-j+1}\right), 1 \leq \beta \leq h-j+1$ in such a way that $L_{i \beta}^{i-j+1} \in \mathcal{M}_{r_{i}, m_{h-\beta+1}}$ is a matrix whose last $r_{i}-r_{h-\beta+i-j+1}$ rows are zero and for $\beta \geq i-j+1$, the rows $n_{p q}, 1 \leq p \leq \beta-i+j, 1 \leq q \leq m_{h-p+1}$ are also zero. (Notice that $Y_{i j}$ follows from $\left.Y_{i-j+1,1}\right)$. 
Proof .- The proof is constructive and consists of eliminating as many parameters of $X$ as possible by using the action of suitable elementary matrices. We shall divide the process into five steps.

(i) Since $\operatorname{rank} X_{i i}=s_{i}$, one has that $\operatorname{rank} Z_{i \alpha}^{1}=\operatorname{rank} Y_{i \alpha}^{1}=m_{h-\alpha+1}, 1 \leq i \leq h, 1 \leq$ $\alpha \leq h-i+1$. So, in particular for $\alpha=1$, we can find an invertible matrix $Q_{1}$ and a sequence of positive integers $n_{11}<n_{12}<\ldots<n_{1, m_{h}}$ so that $Y_{i 1}^{1} Q_{1}$ is a matrix whose rows $n_{11}, n_{12}, \ldots, n_{1 m_{h}}$ are the unit vectors

$$
e_{j}^{1}=\left(\begin{array}{lll}
0 \cdots 0 & \stackrel{(j}{1} & 0 \cdots 0
\end{array}\right) \in \mathbb{K}^{m_{h}}, \quad 1 \leq j \leq m_{h} .
$$

The positive integers $n_{1 j}, 1 \leq j \leq m_{h}$ correspond to $m_{h}$ linearly independent rows of $Y_{i 1}^{1}$. Then, if we denote $P_{i i}^{(1)}=\operatorname{diag}\left(Q_{1}, I_{s_{i}-m_{h}}\right)$ and $P_{1}=\operatorname{diag}\left(P_{11}^{(1)}, \ldots, P_{h h}^{(1)}\right)$ we have that $P_{1} \in \mathcal{G}_{I}(\underline{s})$ and $X^{(1)}=X P_{1}$ is a matrix of $\mathcal{M}(\underline{r}, \underline{s})$ such that for $1 \leq i \leq k, 1 \leq j \leq h$

$$
X_{i j}^{(1)}=\left(\begin{array}{lll}
Z_{i 1}^{i-j+1} Q_{1} \quad Z_{i 2}^{i-j+1} \cdots Z_{i, h-j+1}^{i-j+1}
\end{array}\right)
$$

where the submatrix of $Z_{i 1}^{1} Q_{1}$ formed by the rows $n_{11}, n_{12}, \ldots, n_{1 m_{h}}$ is the matrix $I_{m_{h}}$.

(ii) For $\beta=2,3, \ldots, h$, let $S_{i \beta}^{1}$ be the submatrix of $Z_{i \beta}^{1}$ formed by the rows $n_{11}, \ldots, n_{1 m_{h}}$. Then, the rows $n_{11}, n_{12}, \ldots, n_{1 m_{h}}$ of $Z_{i \beta}^{1}-Z_{i 1}^{1} Q_{1} S_{i \beta}^{1}$ are all zero for $1 \leq i \leq h-1,2 \leq \beta \leq h-i+1$. This is equivalent to multiplying $X^{(1)}$ by a suitable elementary matrix $P_{2} \in \mathcal{G}_{I I}(s)$. So, $X^{(2)}=X^{(1)} P_{2}$ is a matrix of $\mathcal{M}(\underline{r}, \underline{s})$ such that the rows $n_{11}, n_{12}, \ldots, n_{1 m_{h}}$ of $X_{i i}^{(2)}$ are the vectors $e_{11}^{i}, \ldots, e_{1 m_{h}}^{i}$, respectively, being

$$
e_{1 j}^{i}=\left(\begin{array}{lll}
0 \cdots 0 & \stackrel{(j}{1} & 0 \cdots 0
\end{array}\right) \in \mathbb{K}^{s_{i}}, \quad 1 \leq i \leq h .
$$

That is to say, with the submatrix $I_{m_{h}}$ of $Z_{i 1}^{1}, 1 \leq i \leq h$, we cancel out the elements of $Z_{i \alpha}^{1}$, $\alpha>1$, corresponding to the rows where the submatrix $I_{m_{h}}$ lies in $Z_{i 1}^{1}$.

(iii) Let $X_{i i}^{(2)}=\left(A_{i 1}, A_{i 2}, \ldots, A_{i h-i+1}\right)$, with $A_{i \alpha}$ having size $r_{i} \times m_{h-\alpha+1}, 1 \leq i \leq h$.

Since $X^{(2)} \in \mathcal{M}(\underline{r}, \underline{s})$, we know that

$$
A_{i 2}=\left(\begin{array}{c}
B_{i 2} \\
\mathbf{0}
\end{array}\right)
$$

where $B_{i 2} \in \mathcal{M}_{r_{h-1}, m_{h-1}}$ has zero its $n_{11}, n_{12}, \ldots, n_{1 m_{h}}$ rows and

$$
\operatorname{rank} B_{i 2}=\operatorname{rank} A_{i 2}=m_{h-1} .
$$

So, if $1 \leq n_{21}<n_{22}<\ldots<n_{2 m_{h-1}} \leq r_{h-1}$ are $m_{h-1}$ linearly independent rows of $B_{i 2}$, by an analogous procedure to the one used in part (i) we conclude that there exists $P_{3} \in \mathcal{G}_{I}(\underline{s})$ 
such that $X^{(3)}=X^{(2)} P_{3}$ is a matrix of $\mathcal{M}(\underline{r}, \underline{s})$ such that the submatrix formed by the rows $n_{21}, n_{22}, \ldots, n_{2 m_{h-1}}$ and the columns $m_{h}+1, \ldots, m_{h}+m_{h-1}$ of $X_{i i}^{(3)}$ is the unit matrix $I_{m_{h-1}}$, $1 \leq i \leq h-1$. Notice that $n_{1 i} \neq n_{2 j}$ for every $i, j, 1 \leq i \leq m_{h}, 1 \leq j \leq m_{h-1}$.

Then, we proceed as in part (ii) to obtain a matrix $X^{(4)} \in \mathcal{M}(\underline{r}, \underline{s})$ such that if $X^{(4)}=\left(X_{i j}^{(4)}\right)$, $1 \leq i \leq k, 1 \leq j \leq h$, the rows $n_{11}, n_{12}, \ldots, n_{1 m_{h}}, n_{21}, \ldots, n_{2 m_{h-1}}$ of $X_{i i}^{(4)}$ are unit vectors.

(iv) Going on with this process we will obtain a matrix $X^{\prime}$ such that

(a) $X^{\prime}=X P$ for some $P \in \mathcal{G}(\underline{s})$, so that $X^{\prime} \in \mathcal{M}(\underline{r}, \underline{s})$.

(b) If $X^{\prime}=\left(X_{i j}^{\prime}\right), 1 \leq i \leq k, 1 \leq j \leq h$, and $X_{i i}^{\prime}=\left(L_{i 1}^{1} \quad L_{i 2}^{1} \cdots L_{i h-i+1}^{1}\right), 1 \leq i \leq h$ then, for $1 \leq \alpha \leq h-i+1$

$$
L_{i \alpha}^{1}=\left(\begin{array}{c}
N_{i \alpha}^{1} \\
\mathbf{0}
\end{array}\right) \in \mathcal{M}_{r_{i}, m_{h-\alpha+1}}
$$

with $N_{i \alpha}^{1} \in \mathcal{M}_{r_{h-\alpha+1}, m_{h-\alpha+1}}$ and its rows

$$
n_{11}, \ldots, n_{1 m_{h}}, n_{21}, \ldots, n_{2 m_{h-1}}, \ldots, n_{\alpha-1,1}, \ldots, n_{\alpha-1, m_{h-\alpha+2}}
$$

$\left(n_{0 j}=0\right.$ for all $\left.j\right)$ are zero and its rows $n_{\alpha 1}, \ldots, n_{\alpha m_{h-\alpha+1}}$ are the unit vectors $e_{1}^{\alpha}, \ldots, e_{m_{h-\alpha+1}}^{\alpha}$, respectively,

$$
e_{j}^{\alpha}=\left(\begin{array}{lllllll}
0 & \cdots & 0 & 1 & 0 & \cdots & 0
\end{array}\right) \in \mathbb{K}^{m_{h-\alpha+1}}, \quad 1 \leq j \leq m_{h-\alpha+1}
$$

(v) Finally we are going to use elementary matrices of type $I I I$ in order to make zero as many elements from $X_{i j}^{\prime}$ as possible when $i>j$. For notational convenience matrix $X^{\prime}$ will be called $X$, and according to the above notation the standard block decomposition of $X_{i j}$ is denoted $X_{i j}=\left(\begin{array}{lll}L_{i 1}^{i-j+1} & \cdots & L_{i, h-j+1}^{i-j+1}\end{array}\right)$.

To begin with, we consider the blocks $X_{i, i-1}, 2 \leq i \leq h$. If $P=F_{2} \in \mathcal{G}_{I I I}(s)$ we know that

$$
X P=\left(X_{1}+X_{2} Q_{21}, \ldots, X_{h-1}+X_{h} Q_{h, h-1}, X_{h}\right) .
$$

We will pay attention to the effect of the transformation $X_{21}+X_{22} Q_{21}$ on the original block $X_{21}$. We know, by part (iv) of Proposition 2.5 that blocks $X_{32}+X_{33} Q_{32}, \ldots, X_{h, h-1}+X_{h, h} Q_{h, h-1}$ are modified accordingly.

Bearing in mind that

$$
Q_{21}=\left(\begin{array}{ccccc}
0 & R_{12}^{2} & R_{13}^{2} & \ldots & R_{1 h}^{2} \\
0 & 0 & R_{23}^{2} & \ldots & R_{2 h}^{2} \\
\vdots & & \vdots & \ddots & \vdots \\
0 & 0 & 0 & \ldots & R_{h-1, h}^{2}
\end{array}\right)
$$


we have $X_{22} Q_{21}=\left(\begin{array}{llll}0 & G_{2} & \cdots & G_{h}\end{array}\right)$, where

$$
G_{\beta}=\left(\begin{array}{lll}
L_{21}^{1} & \cdots & L_{2, \beta-1}^{1}
\end{array}\right)\left(\begin{array}{c}
R_{1 \beta}^{2} \\
\vdots \\
R_{\beta-1, \beta}^{2}
\end{array}\right), \quad 2 \leq \beta \leq h
$$

So block $X_{21}=\left(L_{21}^{2} \cdots L_{2 h}^{2}\right)$ is transformed into

$$
\left(\begin{array}{lll}
L_{21}^{2} & L_{22}^{2}+G_{2} \cdots L_{2 h}^{2}+G_{h}
\end{array}\right)
$$

Notice that $L_{21}^{2}$ remains unchanged. Let us analyze block $G_{\beta}$ closely .

Let $S_{\ell j}$ denote the submatrix of $L_{2 \ell}^{1}$ formed by the rows $n_{j 1}, \ldots, n_{j m_{h-j+1}}, 1 \leq \ell \leq \beta-2$, $\ell+1 \leq j \leq \beta-1$. Then :

- $R_{1 \beta}^{2}$ is the submatrix of $G_{\beta}$ formed by its rows $n_{11}, \ldots, n_{1 m_{h}}$.

- $S_{12} R_{1 \beta}^{2}+R_{2 \beta}^{2}$ is the submatrix of $G_{\beta}$ formed by its rows $n_{21}, \ldots, n_{2 m_{h-1}}$ of $G_{\beta}$.

- etc.

- $S_{1 \beta-1} R_{1 \beta}^{2}+\ldots+S_{\beta-2, \beta-1} R_{\beta-2, \beta}^{2}+R_{\beta-1, \beta}^{2}$ is the submatrix of $G_{\beta}$ formed by its rows $n_{\beta-1,1}, \ldots, n_{\beta-1, m_{h-\beta+2}}$.

Thus we can choose $Q_{21}$ so that for $1 \leq i \leq \beta-1,1 \leq j \leq m_{h-i+1}$ the rows $n_{i j}$ of $L_{2 \beta}^{2}$, are zero, $2 \leq \beta \leq h$.

As we have said, the remainder blocks $X_{i, i-1}, 3 \leq i \leq h$, are determined from $X_{21}$ according to part(iv) of Proposition 2.5. In general, for any $i>1$ the block $X_{i 1}$ is transformed into $X_{i 1}+X_{i i} Q_{i 1}$ where $Q_{i 1}$ is the $(i, 1)$ block of $F_{i}$. Then, with the above notation it can be checked that the $\beta$ th column-block of $X_{i 1}+X_{i i} Q_{i 1}$ is

$$
L_{i \beta}^{i}+L_{i 1}^{1} R_{1 \beta}^{i}+\ldots+L_{i, \beta-i+1}^{1} R_{\beta-i+1, \beta}^{i}
$$

and with a similar reasoning to the former case we conclude that the rows $n_{p q}$ of $L_{i \beta}^{i}, 1 \leq p \leq$ $\beta-i+1,1 \leq q \leq m_{h-p+1}$ can be reduced to zero for $\beta \geq i$.

Corollary 3.4 .- The number of parameters of matrix $Y \in \mathcal{M}(\underline{r}, \underline{s})$ in the previous theorem coincide with the dimension of $\operatorname{Inv}(\underline{k} ; \underline{h})$.

Proof The number of parameters of $Y$ is equal to $\sum_{j=1}^{k} \sum_{i=1}^{h} m_{i} r_{i+j-1}$ minus the number of entries of the rows that we made zero or unit vectors. Let us count these entries: 
- In $Z_{1 \beta}^{1}$ we converted $m_{h-\beta+1}$ rows into unit vectors and $m_{h}+\ldots+m_{h-\beta+2}$ rows into zero. So, the number of parameters eliminated in $Z_{1 \beta}^{1}$ is $m_{h-\beta+1}\left(m_{h}+\ldots+m_{h-\beta+1}\right)=$ $m_{h-\beta+1} s_{h-\beta+1}$ and hence the number of parameters removed from $X_{11}$ is

$$
\sum_{\beta=1}^{h} m_{h-\beta+1} s_{h-\beta+1}=\sum_{i=1}^{h} m_{i} s_{i} .
$$

- In $Z_{2 \beta}^{2}$ we converted into zero $m_{h}+\ldots+m_{h-\beta+2}=s_{h-\beta+2}$ rows, so that the number of parameters removed from $X_{21}$ is

$$
\sum_{\beta=1}^{h} m_{h-\beta+1} s_{h-\beta+2}=\sum_{i=1}^{h} m_{i} s_{i+1}
$$

- etc.

- In general, the number of parameters removed from $X_{j 1}$ is

$$
\sum_{i=1}^{h} m_{i} s_{i+j-1}, \quad 1 \leq j \leq k .
$$

Consequently, the number of parameters of $Y$ is

$$
\sum_{j=1}^{k} \sum_{i=1}^{h} m_{i}\left(r_{i+j-1}-s_{i+j-1}\right) .
$$

which coincides with the dimension of $\operatorname{Inv}(\underline{k} ; \underline{h})$ as Corollary 2.9 shows.

Definition 3.5 With the notation of the previous theorem we will say that $Y$ is a reduced form of $X$, and the set of positive integers $\left\{n_{i j}: 1 \leq i \leq h, 1 \leq j \leq m_{h-i+1}\right\}$ will be said to be an admissible set of indices for $X$.

Example 3.6 If in Example 2.2 we assume that $x_{2} \neq 0$, we can get two reduced form of $X$ :

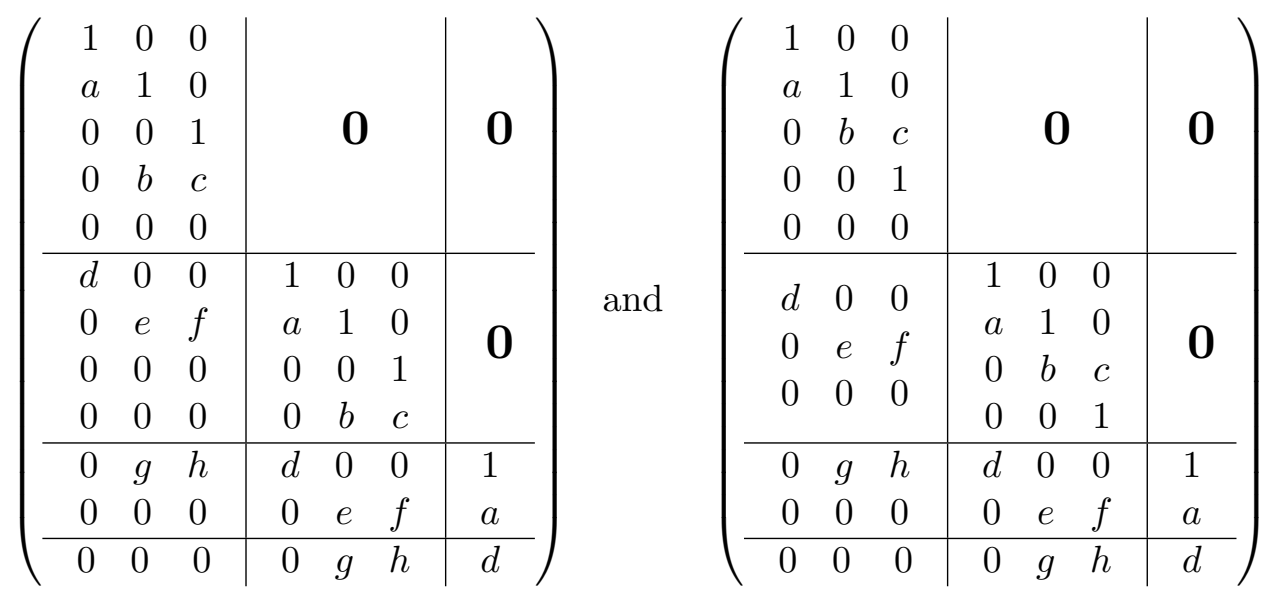


with $n_{11}=1, n_{21}=1, n_{22}=3$ and $n_{11}=1, n_{21}=2, n_{22}=4$ as admissible set of indices, respectively. And if we assume that $t_{1} \neq 0$, then the reduced forms of $X$ are

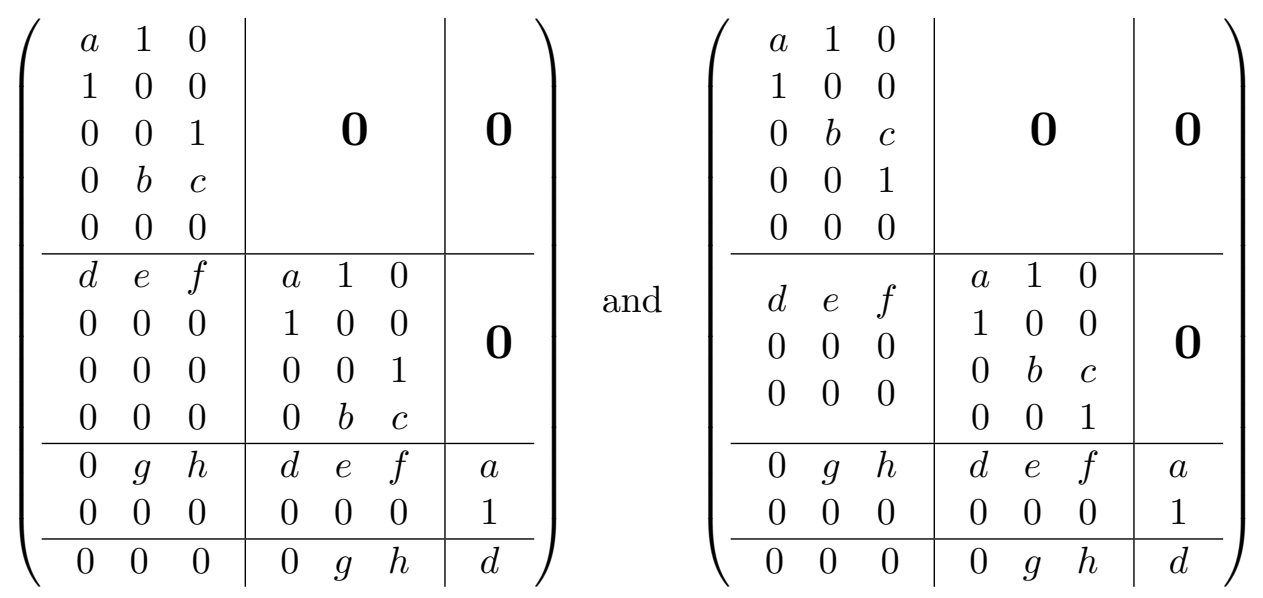

with $n_{11}=2, n_{21}=1, n_{22}=3$ and $n_{11}=2, n_{21}=1, n_{22}=4$ as admissible set of indices, respectively. Indeed, these are the only possible cases.

Remark 3.7 .-The following remarks are intended to clarify a little more the structure of the reduced forms:

(i) If $k>h$ then for $1 \leq j \leq h<i \leq k$ the blocks $X_{i j}$ of $X$ and of any of its possible reduced forms are the same.

(ii) Taking into account how an admissible set of indices for $X$ has been obtained, it is clear that the condition of "being an admissible set" is generic. That is to say, if $\left\{n_{i j}\right\}$ is an admissible set of indices for $X$, then it is also an admissible set of indices for small enough perturbations of $X$.

(iii) The structure of the off-diagonal blocks of reduced forms is a direct consequence of the structure of the diagonal blocks. In other words, if $P \in \mathcal{G}(\underline{s})$ is a matrix such that the diagonal blocks of $X P$ have the structure described in part (iii) of Theorem 3.3 then there is also a matrix $Q \in \mathcal{G}(\underline{s})$ so that $X P$ and $(X P) Q$ have the same diagonal blocks and for $i>j$ the blocks $((X P) Q)_{i j}$ have the form described in part 4 of the theorem.

A simple but interesing consequence of Theorem 3.3 is the following

Corollary 3.8 Any matrix of $\mathcal{G}(\underline{s})$ is a product of a finite number of elementary matrices.

Proof . If $P \in \mathcal{G}(\underline{s})$ then $P \in \mathcal{M}(\underline{s}, \underline{s})$ and an admissible set of indices for $P$ is $n_{i j}=$ $m_{h}+\ldots+m_{h-i+2}+j, 1 \leq j \leq m_{h-i+1}, 1 \leq i \leq h$. In other words, a reduced for of $P$ is $I_{d}$. 
But we have seen in the proof of Theorem 3.3 that any matrix of $\mathcal{M}(\underline{r}, \underline{s})$ can be brought to a reduced form by means of elementary matrices. Then

$$
I_{d}=P Q
$$

for some $Q \in \mathcal{G}(\underline{s})$ which is a product of a finite number of elementary matrices. Now, the inverse of an elementary matrix is an elementary matrix of the same type, as one can easily check, and so the corollary follows.

In order to prove that the set of reduced forms (depending on the admissible indices $n_{i j}$ ) constructed in the previous theorem defines an atlas of coordinate charts for $\mathcal{M}(\underline{r}, \underline{s}) / \mathcal{G}(\underline{s})$, we need the following two lemmas.

Lemma 3.9 Let $X \in \mathcal{M}(\underline{r}, \underline{s})$ and $Q \in \mathcal{G}(\underline{s})$. If $\left\{n_{i j}, 1 \leq i \leq h, 1 \leq j \leq m_{h-i+1}\right\}$ is an admissible set of indices for $X$, it is also an admissible set of indices for $X Q$.

Proof. Let $Y=X P$ be a reduced form for $X$ corresponding to the given set of indices. Then $X Q=Y P^{-1} Q$ for some $P \in \mathcal{G}(\underline{s})$ and this means that we can assume without lost of generality that $X$ is in reduced form $Y$. Then with the notation in Proposition 2.7 and Theorem 3.3 we have that

$$
(Y Q)_{11}=\left(L_{11}^{1} T_{1 j}^{1}+L_{12}^{1} T_{2 j}^{1}+\ldots+L_{1 j}^{1} T_{j j}^{1}\right)_{1 \leq j \leq h}
$$

We will prove by induction on $j=1,2, \ldots, h$ that there is a matrix $P \in \mathcal{G}(\underline{s})$ such that if $Q P=\left(S_{i j}\right)_{1 \leq i, j \leq h}$ (we are using the notation of Proposition 2.7) then for $1 \leq t \leq j$ the rows

$$
n_{11}, \ldots, n_{1 m_{h}}, n_{21}, \ldots, n_{2 m_{h-1}}, \ldots, n_{t-11}, \ldots, n_{t-1 m_{h-t}}
$$

of $L_{11}^{1} S_{1 t}^{1}+L_{12}^{1} S_{2 t}^{1}+\ldots+L_{1 t}^{1} S_{t t}^{1}$ are zero and its rows $n_{t 1}, \ldots, n_{t m_{h-t+1}}$ are unit vectors. The case $j=h$ proves the lemma.

Assume that $j=1$ and recall that since $\left\{n_{i j}: 1 \leq i \leq h, 1 \leq j \leq m_{h-i+1}\right\}$ is an admissible set of indices we have that the rows $n_{11}, \ldots n_{1 m_{h}}$ of $L_{11}^{1}$ are linearly independent. As $T_{11}^{1}$ is invertible, the same rows of $L_{11}^{1} T_{11}^{1}$ are linearly independent too. If we set $T_{i}=\operatorname{diag}\left(\left(T_{11}^{1}\right)^{-1}, I_{m_{h-1}+\ldots, m_{h-i+1}}\right), 1 \leq i \leq h$ and $P=\operatorname{diag}\left(T_{1}, \ldots, T_{h}\right)$ then $S_{11}^{1}=I_{m_{h}}$ and $L_{11}^{1} S_{11}^{1}=L_{11}^{1}$.

Now let $j>1$. By the induction hypothesis there is a matrix $\tilde{P} \in \mathcal{G}(\underline{s})$ such that if $Q \tilde{P}=$ $\left(\tilde{S}_{i j}\right)_{1 \leq i, j \leq h}$ then for $1 \leq t \leq j-1$ the rows $\left\{n_{i \alpha}: 1 \leq i \leq t-1,1 \leq \alpha \leq m_{h-i+1}\right\}$ of $L_{11}^{1} \tilde{S}_{1 t}^{1}+L_{12}^{1} \tilde{S}_{2 t}^{1}+\ldots+L_{1 t}^{1} \tilde{S}_{t t}^{1}$ are zero and its rows $n_{t 1}, \ldots, n_{t m_{h-t+1}}$ are unit vectors. By using the same procedure as in Theorem 3.3 (see step (ii)) we can find an elementary matrix $P_{1} \in \mathcal{G}_{I I}(\underline{s})$ such that if $\left(Y Q \tilde{P} P_{1}\right)_{11}=\left(A_{1 j}\right)_{1 \leq j \leq h}$ is the standard block decomposition of the $(1,1)$-block, then for $t=1,2, \ldots, j$ the rows $\left\{n_{i \alpha}: 1 \leq i \leq t-1,1 \leq \alpha \leq m_{h-i+1}\right\}$ of $A_{1 t}$ are zero and for $t=1,2, \ldots, j-1$ its rows $\left\{n_{t \alpha}: 1 \leq \alpha \leq m_{h-t+1}\right\}$ are unit vectors. Thus 
we only have to show that the rows $n_{j 1}, \ldots, n_{j m_{m-j+1}}$ of $A_{1 j}$ are linearly independent. Put $Q \tilde{P} P_{1}=\left(S_{i j}\right)_{1 \leq i, j \leq h}$, then

$$
A_{1 j}=L_{11}^{1} S_{1 j}^{1}+\ldots L_{1 j}^{1} S_{j j}^{1} .
$$

Bearing in mind that the rows $n_{11}, \ldots, n_{1 m_{h}}$ of $A_{1 j}$ are zero, that the same rows in $L_{12}^{1}, \ldots, L_{1 j}^{1}$ are zero as well and that these rows in $L_{11}^{1}$ are unit vectors we conclude that $S_{1 j}^{1}=0$. From this and the fact that rows $n_{21}, \ldots, n_{2 m_{h-1}}$ of $A_{1 j}$ and $L_{13}^{1}, \ldots, L_{1 j}^{1}$ are zero and these rows in $L_{12}^{1}$ are unit vectors we get $S_{2 j}^{1}=0$. Going on this way it follows that $S_{1 j}^{1}=\ldots S_{j-1 j}^{1}=0$. Finally, as $S_{j j}^{1}$ is invertible and the rows $n_{j 1}, \ldots, n_{j m_{h-j+1}}$ of $L_{1 j}^{1}$ are unit vectors, we conclude that these rows in $A_{1 j}$ are linearly independent as desired.

So, we can choose for all matrices in the orbit $X \mathcal{G}(\underline{s})$ the same set of indices. Next lemma shows that the corresponding reduced matrices are the same.

Lemma 3.10 Let $Y$ and $\bar{Y}$ be matrices of $\mathcal{M}(\underline{r}, \underline{s})$ in reduced form with the same set of indices $n_{i j}, 1 \leq i \leq h, 1 \leq j \leq m_{h-i+1}$. If there is a matrix $P \in \mathcal{G}(\underline{s})$ such that $\bar{Y}=Y P$ then $P=I_{d}$.

Proof. Let us denote by $L_{i j}$, resp. $\bar{L}_{i j}$, the standard block decomposition of $Y$ and $\bar{Y}$, respectively. It is enough to compare the corresponding blocks in $Y_{1}$ and $\bar{Y}_{1} P$. Then taking into account where the rows with unit vectors and zeros are placed in $Y$ and $\bar{Y}$ as well as the partition into blocks of $P$ given in Proposition 2.7, the lemma follows.

We finally define the coordinate charts of $\mathcal{M}(\underline{r}, \underline{s}) / \mathcal{G}(\underline{s})$. From the above lemmas we conclude that to every point of $\mathcal{M}(\underline{r}, \underline{s}) / \mathcal{G}(\underline{s})$ we can associate a matrix in reduced form. This correspondence is defined as follows: take a representative $X \in \mathcal{M}(\underline{r}, \underline{s})$ of the point of $\mathcal{M}(\underline{r}, \underline{s}) / \mathcal{G}(\underline{s})$ and according to the process described in Theorem 3.3 obtain a reduced matrix $Y$. This matrix $Y$ depends only on the set of indices $n_{i j}$ that we have chosen along the process, but not on the representative $X$. Furthermore, according to Remark 3.7 (ii), there is an open neighborhood of $X$ such that for every matrix in this neighborhood we can choose the same admissible set of indices $n_{i j}$.

Conversely, it is clear that if $\left\{n_{i j}, 1 \leq i \leq h, 1 \leq j \leq m_{h-i+1}\right\}$ is a set of pairwise different positive integers such that for $1 \leq i \leq h$,

$$
1 \leq n_{i 1}<n_{i 2}<\ldots<n_{i m_{h-i+1}} \leq r_{h-i+1}
$$

there exists a matrix $X \in \mathcal{M}(\underline{r}, \underline{s})$ having the above set as an admissible set of indices.

So, if we denote $\Lambda$ the set of indices $I=\left(n_{i j}\right), 1 \leq i \leq h, 1 \leq j \leq m_{h-i+1}$ verifying the above conditions and $\mathcal{U}_{I}$ is the set of matrices $X \in \mathcal{M}(\underline{r}, \underline{s})$ such that $I$ is admissible for $X$, one has that $\left\{\mathcal{U}_{I} ; I \in \Lambda\right\}$ is an open covering of $\mathcal{M}(\underline{r}, \underline{s})$. Hence, if $\pi: \mathcal{M}(\underline{r}, \underline{s}) \longrightarrow \mathcal{M}(\underline{r}, \underline{s}) / \mathcal{G}(\underline{s})$ is the natural projection, $\left\{\widetilde{\mathcal{U}}_{I}=\pi\left(\mathcal{U}_{I}\right) ; I \in \Lambda\right\}$ is an open covering of $\mathcal{M}(\underline{r}, \underline{s}) / \mathcal{G}(\underline{s})$. 
Finally, put $N=\operatorname{dim} \operatorname{Inv}(\underline{k} ; \underline{h})$, take $I \in \Lambda$ and define the mapping

$$
\varphi_{I}: \mathcal{U}_{I} \longrightarrow \mathbb{K}^{N}
$$

in the following way: for every $X \in \mathcal{U}_{I}, \varphi_{I}(X)$ is the point in $\mathbb{K}^{N}$ defined by the parameters of the reduced form of $X$, corresponding to $I, Y_{I}$, given in a certain order. Taking into account the way that we obtained $Y_{I}$ it turns out that $\varphi_{I}$ is differentiable. The mapping $\varphi_{I}$ induces a mapping

$$
\theta_{I}: \widetilde{\mathcal{U}}_{I} \longrightarrow \mathbb{K}^{N}
$$

We can now state the main result of this section.

Theorem 3.11 With the above notation $\theta_{I}$ is a diffeomorphism and $\left\{\widetilde{U}_{I}, I \in \Lambda\right\}$ is a coordinate atlas of $\mathcal{M}(\underline{r}, \underline{s}) / \mathcal{G}(\underline{s})$.

Proof. It is clear that $\theta_{I}$ is bijective. The fact that $\theta_{I}$ is differentiable follows from the existence of local sections for $\pi: \mathcal{M}(\underline{r}, \underline{s}) \longrightarrow \mathcal{M}(\underline{r}, \underline{s}) / \mathcal{G}(\underline{s})$ and one checks easily that $\theta_{I}^{-1}$ is also differentiable.

Remark 3.12 (i) As $\mathcal{M}(\underline{r}, \underline{s}) / \mathcal{G}(\underline{s})$ has been identified with the homogeneous space Inv $(\underline{k}, \underline{h})$, a coordinate atlas of $\operatorname{Inv}(\underline{k}, \underline{h})$ has been obtained.

(ii) Since the matrices $Y_{I}$ determine the coordinates of every $\pi(X), X \in \mathcal{U}_{I}$, we will say that $Y_{I}$ defines a coordinate system in $\operatorname{Inv}(\underline{k} ; \underline{h})$.

Example 3.13 Going back to Example 3.6 and taking into account the above remark we have that a coordinate atlas of $\operatorname{Inv}(\underline{k}, \underline{h})$ for $\underline{k}=(4,3,2,2,1)$ and $\underline{h}=(3,2,2)$ is defined by the four sets of reduced matrices described in the example.

\section{An example}

As said in the introduction, having a coordinate chart of the conditioned invariant subspaces may have some useful applications in Control Theory. Namely, in those instances where some computations involving such invariant subspaces are required. As an example we will consider the Disturbace Decoupling Problem (DDP). It is well-known that this problem consists in finding a state feedback $F$ to be performed on the system

$$
\left\{\begin{array}{l}
\dot{x}(t)=A x(t)+B u(t)+Q q(t) \\
y(t)=C x(t)
\end{array}\right.
$$


in order the closed-loop system

$$
\left\{\begin{array}{l}
\dot{x}(t)=(A+B F) x(t)+B u(t)+Q q(t) \\
y(t)=C x(t)
\end{array}\right.
$$

to have an output $y(t)$ that does not depend on the disturbance $q(t)$, for any initial state. We assume that $A \in \mathcal{M}_{n}, B \in \mathcal{M}_{n, m}, Q \in \mathcal{M}_{n, s}$ and $C \in \mathcal{M}_{p, n}$. In addition we assume also that $Q$ and $C$ are full rank matrices.

A solution to this problem can be found, for example, in [W, Th. 4.2] where the following result is proved: the DDP has a solution if and only if

$$
\Im(A, B ; \operatorname{Ker} C) \supset \operatorname{Im} Q
$$

where $\Im(A, B ; \operatorname{Ker} C)$ is the unique maximal $(A, B)$-invariant subspace contained in $\operatorname{Ker} C$. The question that we want to address here is to find the set of full rank output matrices $C \in \mathcal{M}_{p, n}$ such that the ouput $y(t)=C x(t)$ is independent of $q(t)$. In particular this allows us to obtain the maximal possible rank for such a matrix $C$ to verify this property. As pointed out in $[\mathrm{WE}]$ this is interesting, for example, in order to determine limited order observers. So we are looking for matrices $C \in \mathcal{M}_{p, n}^{*}$ such that there is an $(A, B)$-invariant subspace, $\mathcal{S}$, satisfying

$$
\operatorname{Im} Q \subset \mathcal{S} \subset \operatorname{Ker} C
$$

By duality this is equivalent to finding matrices $C$ for which there is a $\left(B^{T}, A^{T}\right)$-invariant subspace, $\mathcal{X}\left(=\mathcal{S}^{\perp}\right)$, so that

$$
\operatorname{Im} C^{T} \subset \mathcal{X} \subset(\operatorname{Im} Q)^{\perp}
$$

If $(\operatorname{Im} Q)^{\perp}$ is itself $\left(B^{T}, A^{T}\right)$-invariant, $\operatorname{dim}(\operatorname{Im} Q)=s$ and $(\operatorname{Im} Q)^{\perp}=[S]$ then $C^{T}=S X$ is one of the desired matrices for any choice of $X \in \mathcal{M}_{n-s, p}^{*}$. If $(\operatorname{Im} Q)^{\perp}$ is not $\left(B^{T}, A^{T}\right)$ invariant we can find, with the help of the coordinate charts introduced in the previous section (and provided that the system is controlable), all $\left(B^{T}, A^{T}\right)$-invariant subspaces, $\mathcal{X}$, of any given dimension contained in $(\operatorname{Im} Q)^{\perp}$. We will show with one example that this reduces to solve a linear system of algebraic equations. Once we have one of such invariant subspaces, say $\mathcal{S} \subset(\operatorname{Im} Q)^{\perp}$, the matrices $C$ for which $\operatorname{Im} C^{T} \subset \mathcal{X}$ are obtained as above: $C^{T}=S X$ where $\mathcal{X}=[S]$ and $X$ is any $d \times p$ full column rank matrix, $d$ being the dimension of $\mathcal{X}$. The restriction on the controllability of the systems comes from the fact that we only have a parameterization of the conditioned invariant subspaces in the observability case.

Let us show an example of the method to obtain the $\left(B^{T}, A^{T}\right)$-invariant subspaces contained in $(\operatorname{Im} Q)^{\perp}$. Let $n=12, m=4$ and $s=1$ (scalar disturbance). Let also the controllability indices of $(A, B)$ (or observability indices of $\left(B^{T}, A^{T}\right)$ ) be $\underline{k}=(5,4,2,1)$. As $s=1$ and $Q$ has full rank we can assume that $\operatorname{Im} Q$ is (generically) of the following form:

$$
\operatorname{Im} Q=\left[\left(\begin{array}{c}
-a_{1} \\
-a_{2} \\
\vdots \\
-a_{11} \\
1
\end{array}\right)\right]
$$


and

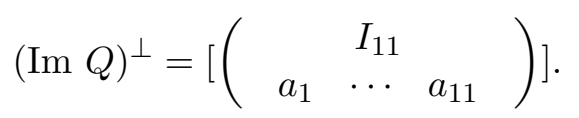

If we are looking for $\left(B^{T}, A^{T}\right)$-invariant subspaces $\mathcal{X} \subset(\operatorname{Im} Q)^{\perp}$ of dimension $d=7$, we must find all possible partitions $\underline{h}$ compatible with $\underline{k}$ (i. e. $k_{i} \geq h_{i}, i=1, \ldots, 4$ ) in order to get the observability indices of the restrictions of $\left(B^{T}, A^{T}\right)$ to $\mathcal{X}$. One of such partitions is $\underline{h}=(3,3,1)$. It might happen that there be no $\left(A^{T}, B^{T}\right)$-invariant subspaces, $\mathcal{X}$, whose restrictions have $(3,3,1)$ as observability indices and such that $\mathcal{X} \subset(\operatorname{Im} Q)^{\perp}$. As we will see soon, this depends on the compatibility of certain linear system. If this is compatible, its solution we will provide us with the necessary elements to construct a basis for a subspace which is both $\left(B^{T}, A^{T}\right)$-invariant and a subspace of $(\operatorname{Im} Q)^{\perp}$.

First, if $\mathcal{X}$ is $\left(B^{T}, A^{T}\right)$-invariant and its restricctions has $\underline{h}=(3,3,1)$ as observability indices, it must belong to (at least) one of the coordinate charts of $\operatorname{Inv}(\underline{k}, \underline{h})$ (recall that the coordinate charts form a covering of this manifold and that each one of them is an open and dense set; and so "almost al" ( $\left.B^{T}, A^{T}\right)$-invariant subspaces belongs to each chart). The coordinate charts for the case that we are considering here were obtained in Example 3.6. Let us assume that $\mathcal{X}$ belongs to the coordinate chart determined by the indices $n_{11}=1, n_{21}=2$ and $n_{22}=3$ (if $\mathcal{X}$ belongs to any other coordinate charts then we would proceed similarly). Thus

$$
\left.\mathcal{X}=\left[\begin{array}{ccc|ccc|c}
1 & 0 & 0 & & & \\
x_{1} & 1 & 0 & & & \\
0 & 0 & 1 & & \mathbf{0} & \mathbf{0} \\
0 & x_{3} & x_{6} & & & & \\
0 & 0 & 0 & & & & \\
\hline x_{2} & 0 & 0 & 1 & 0 & 0 & \\
0 & x_{4} & x_{7} & x_{1} & 1 & 0 & \mathbf{0} \\
0 & 0 & 0 & 0 & 0 & 1 & \\
0 & 0 & 0 & 0 & x_{3} & x_{6} & \\
\hline 0 & x_{5} & x_{8} & x_{2} & 0 & 0 & 1 \\
0 & 0 & 0 & 0 & x_{4} & x_{7} & x_{1} \\
\hline 0 & 0 & 0 & 0 & x_{5} & x_{8} & x_{2}
\end{array}\right)\right] .
$$

On the other hand $\mathcal{X} \subset(\operatorname{Im} Q)^{\perp}$ is equivalent to the existence of a matrix $Y \in \mathcal{M}_{11,7}^{*}$ such 
that

$$
\left(\begin{array}{ccc|ccc|c}
1 & 0 & 0 & & & & \\
x_{1} & 1 & 0 & & & & \mathbf{0} \\
0 & 0 & 1 & & \mathbf{0} & & \\
0 & x_{3} & x_{6} & & & & \\
0 & 0 & 0 & & & & \\
\hline x_{2} & 0 & 0 & 1 & 0 & 0 & \\
0 & x_{4} & x_{7} & x_{1} & 1 & 0 & \mathbf{0} \\
0 & 0 & 0 & 0 & 0 & 1 & \\
0 & 0 & 0 & 0 & x_{3} & x_{6} & \\
\hline 0 & x_{5} & x_{8} & x_{2} & 0 & 0 & 1 \\
0 & 0 & 0 & 0 & x_{4} & x_{7} & x_{1} \\
\hline 0 & 0 & 0 & 0 & x_{5} & x_{8} & x_{2}
\end{array}\right)=\left(\begin{array}{ccc} 
\\
a_{1} & \cdots & a_{11}
\end{array}\right) Y=\left(\begin{array}{c}
Y \\
a Y
\end{array}\right) .
$$

Hence

$$
Y=\left(\begin{array}{ccc|ccc|c}
1 & 0 & 0 & & & & \\
x_{1} & 1 & 0 & & & & \\
0 & 0 & 1 & & \mathbf{0} & & \mathbf{0} \\
0 & x_{3} & x_{6} & & & & \\
0 & 0 & 0 & & & & \\
\hline x_{2} & 0 & 0 & 1 & 0 & 0 & \\
0 & x_{4} & x_{7} & x_{1} & 1 & 0 & \mathbf{0} \\
0 & 0 & 0 & 0 & 0 & 1 & \\
0 & 0 & 0 & 0 & x_{3} & x_{6} & \\
\hline 0 & x_{5} & x_{8} & x_{2} & 0 & 0 & 1 \\
0 & 0 & 0 & 0 & x_{4} & x_{7} & x_{1}
\end{array}\right) \text { and }\left(\begin{array}{ccccccc}
0 & 0 & 0 & 0 & x_{5} & x_{8} & x_{2}
\end{array}\right)=a Y
$$

The existence of matrix $Y$ is then equivalent to the compatibility of the following system:

$$
\left.\begin{array}{l}
a_{1}+a_{2} x_{1}+a_{6} x_{2}=0 \\
a_{2}+a_{4} x_{3}+a_{7} x_{4}+a_{10} x_{5}=0 \\
a_{3}+a_{4} x_{6}+a_{7} x_{7}+a_{10} x_{8}=0 \\
a_{6}+a_{7} x_{1}+a_{10} x_{2}=0 \\
a_{7}+a_{9} x_{3}+a_{11} x_{4}=x_{5} \\
a_{8}+a_{9} x_{6}+a_{11} x_{7}=x_{8} \\
a_{10}+a_{11} x_{1}=x_{2}
\end{array}\right\}
$$

Notice that this is a linear system of $7=s \cdot d$ equations with $8=\operatorname{dim} \operatorname{Inv}(\underline{k}, \underline{h})$ unknowns. Generically it has a solution depending on 1 parameter. The solutions of this system provide the parameters to form bases of $\left(B^{T}, A^{T}\right)$-invariant subspaces contained in $(\operatorname{Im} Q)^{\perp}$, whose restrictions have $\underline{h}=(3,3,1)$ as observability indices and belong to the coordinate chart determined by the indices $n_{11}=1, n_{21}=2$ and $n_{22}=3$. To obtain bases of subspaces belonging to different coordinate charts we should solve similar systems with the same number of equations and unknowns. This means that, generically, either we would find $\left(B^{T}, A^{T}\right)$-invariants subspaces 
contained in $(\operatorname{Im} Q)^{\perp}$ in every coordinate chart or we would not find them in any coordinate chart.

We finally remark that, again generically, the existece of solution of these linear systems depends only on the numbers $s, d$ and $\operatorname{dim} \operatorname{Inv}(\underline{k}, \underline{h})$. Such a system is generically compatible if and only if

$$
s d \leq \operatorname{dim} \operatorname{Inv}(\underline{k}, \underline{h}) .
$$

Notice that $\operatorname{dim} \operatorname{Inv}(\underline{k}, \underline{h})$ does not depend only on $d$ but also on $\underline{k}$ and $\underline{h}$ (see Corollary 2.9). But, in any case, this formula can be easily implemented in a computer in order to check whether or not it holds for every particular case.

\section{Conclusions}

In this paper the problem of parameterize the smooth manifold of $(C, A)$-conditioned invariant subspaces of observable systems with given observability indices for the restriction has been considered. The parameterization is done through an atlas of coordinate charts by identifying the manifold with an homogeneous space. These coordinate charts are useful in those instances where some computations involving invariant subspaces are required. An example of this situation related to the Disturbance Decoupling Problem is presented.

\section{References}

[BM] G. Basile, G. Marro, Controlled and Conditioned Invariants in Linear System Theory Prentice Hall, Englewood Clifs, New Jersey, 1991.

[B] P. Brunovsky, Classification of Linear Controllable Systems. Kybernetika. (Praga), 3 (6), 173-188, 1970.

[FP] J. Ferrer, F. Puerta, Similarity of non-everywhere defined Linear Maps. Linear Algebra Appl., 170, 27-55, 1992.

[FPP1] J. Ferrer, F. Puerta, X. Puerta, Differentiable structure of the set of the controllable $(A, B)^{t}$-invariant subspaces. Linear Algebra Appl., 275-276, 161-177, 1998.

[FPP2] J. Ferrer, F. Puerta, X. Puerta, Stratification of the set of general $(A, B)$-invariant subspaces. Preprint. 1999.

[FH] P. A. Fuhrman, U. Helmke, On Conditioned Invariant Subspaces and Observer Theory. Preprint CIM n.1, 1998.

[1] I. Gohberg, P. Lancaster, L. Rodman. Invariant Subspaces of Matrices with Applications, Wiley, New York, 1986. 
[HS] U. Helmke, M. Shayman, The biflag manifold and the fixed point set of a nilpotent transformation on the flag manifold, Lin. Alg. Appl. 92, 125-159, 1987.

[HMP] D. Hinrichsen, H. F. Münzner, D. Prätze-Wolters, Parametrization of $(C, A)$-invariant subpaces. Systems \& Control Letters, 1, 192-199, 1981.

[PH] X.Puerta, U.Helmke, The topology of the set of conditioned invariant subspaces System and Control Letters, 40,97-105, 2000.

[WE] M.E. Warren, A. E. Eckberg JR. On the Dimensions of Controllability Subspaces: A Characterization via Polynomial Matrices and Kronecker Invariants SIAM J. Control 13(2), 434-445, 1975.

[W] W. M. Wonham, Linear Multivariable Control: A Geometrical Approach. Springer-Verlag, New York, 1984. 